\title{
Synthesis of 4-Methylene-2-cyclohexenones and Their Aromatization Reaction toward para-Methoxylmethyl Anisole Derivatives
}

\author{
Jeong Mì Kỉm, Ka Young Lee, and Jae Nyoung Kim \\ Department of Chemistry and Institute of Basic Science, Chonnam National Lniversity, Guangin 500-757, Korea \\ Received November 24, 2003
}

Key Words : 4-Methylene-2-cyclohexenones, Aromatization. Methoxymethyl anisoles, Baylis-I Iillman acetates

Recently, we have reported on the synthesis of anisole derivatives from 4-alkylidene-2-cyclohexene-l-ones with iodine in methanol. ${ }^{3}$ 4-Alkylidene-2-cyclohexene-l-ones could be synthesized from the reaction of Baylis-Hillman acetates and 2,4-pentanedione according to the reported procedure by Chamakh and Amri, ${ }^{2}$

We and other groups have reported the selective introduction of nucleophiles at the secondary benzylic position of the Baylis-Hillman acetates via the corresponding DABCO salts." Thus, we envisioned that we could prepare 4-methylene-2-cyclohexenone skeleton and para-methoxymethyl anisoles by combining the DABCO salt concept and the aromatization reaction with iodine in methanol. ${ }^{1.5}$ Suitably substituted anisoles are useful as the starting materials for the fragrances, dyes and pesticides, as antioxidants in oils and fats, or as stabilizers of plastics." Moreover, paramethoxymethyl anisoles have been used for the kinetic acetalization of diol or amino alcohol systems in the presence of $D D Q^{5}$ during the synthesis of (+)-FR900482, ${ }^{5 \text { t }}$ taxotere side chain, ${ }^{{ }_{c}}$ cyclopropyl lactone oxylipins, ${ }^{5 \mathrm{~d}}$ and erythromycin A. ${ }^{\text {s. }}$

Our synthetic scheme for the synthesis of 4-methylene-2cyclohexenone skeleton and para-methoxymethyl anisole is shown in Scheme 1 by using $4 \mathbf{a}$ and $5 \mathbf{a}$ as the representative examples. The reaction of the Baylis-Hillman acetate $\mathbf{I a}$ and $\mathrm{DABCO}$ in aqueous $\mathrm{THF}$ gave the corresponding $\mathrm{DABCO}$ salt $2 \mathbf{a}$ instantaneously as reported. ${ }^{3}$ The reaction of the DABCO salt 2a and 2,4-pentanedione afforded the intermediate $3 \mathbf{a}$ in moderate yield (44\%). During the synthesis of 3a, some side products were produced (including dihydropyran skeleton), ${ }^{6}$ which diminished the yield of $\mathbf{3 a}$. With the compound $3 \mathbf{a}$ in our hand, we examined the next conversion toward 4-methylene-2-cyclohexeneone $4 \mathbf{a}$ by using the Amri's conditions $\left(\mathrm{K}_{2} \mathrm{CO}_{3} \text { in ethanol }\right)^{-}$and we could prepare 4-methylene-2-cyclohexenone $4 a$ in good yield (72\%). ${ }^{7}$ The synthesis of 4 a was tried by using the modified conditions in order to improve the yield of $4 \mathbf{a}$ as follows. In situ generation of the DABCO salt $2 \mathbf{a}$ in ethanol and the following reaction with 2,4-pentanedione in the presence of $\mathrm{K}_{2} \mathrm{CO}_{3}$ gave $4 \mathbf{a}$ in a similar isolated yield (47\%) (Scheme 1). ${ }^{8}$ The use of ethyl acetoacetate instead of 2,4-pentanedione also gave 4a in lower yield (not shown in Scherne 1). The structure of $4 \mathbf{a}$ was confirmed as shown in Table 1 by NOF: experiments. As an example, irradiation of the methyl group $(\delta-2.11 \mathrm{ppm})$ of 4 a showed increments of the proton at the 2-position ( $\delta-6.00 \mathrm{ppm})$ and one of the methylene protons $(\delta-5.51 \mathrm{ppm})$ in 0.6 and $0.7 \%$, respectively. $\Lambda$ s the final step, the reaction of 4 a and iodine (1.1 equiv.) in methanol $\left(40-50{ }^{\circ} \mathrm{C}\right)$ aflorded methoxymethyl anisole $5 \mathrm{a}$ in good yicld $(84 \%)$." Similarly, $5 c$ and $5 e$ were synthesized in good yiclds from $4 b$ and 4c. $\Lambda$ s shown in Table 1 , the use of ethanol as the solvent afforded the ethoxymethyl phenetole derivatives

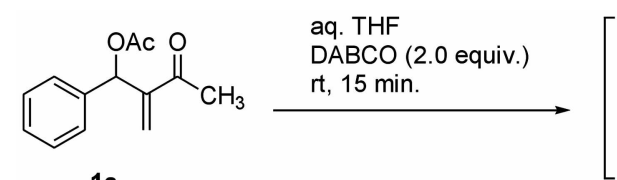

1a

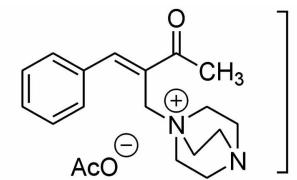

2a

rt, $15 \mathrm{~min}$

reflux, 30 min, $47 \%$
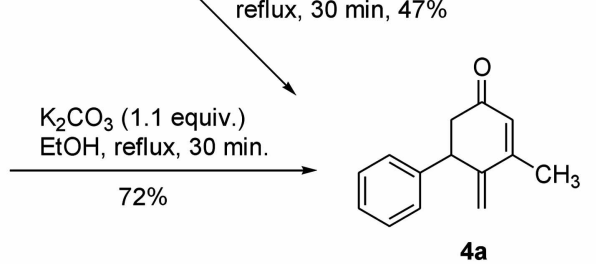

$4 a$ 2,4-pentanedione ( 1.1 equiv.) rt, $24 \mathrm{~h}, 44 \%$

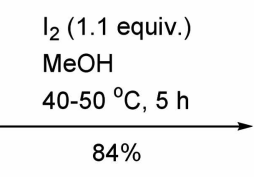

Scheme 1<smiles>C#CC(C#C)C(C(=C)C(C)=O)c1ccccc1</smiles>

$3 a$

\footnotetext{
"Coresponding Author. Phonc: +82-62-530-3381, c-mail: kimjn@ 0 chonnam.ac.kr
} 
Table 1. Synthesis of 4-methylene-2-cyclohexenones 4, 4-methoxymethyl anisoles $\mathbf{5 a}, \mathbf{5 c}$, 5e, and 4-ethoxymethyl phenetoles $\mathbf{5 b}$, $\mathbf{5 d}, \mathbf{5 f}$

\begin{tabular}{|c|c|c|c|c|c|}
\hline F.ntry & B-II acetate 1 & Conditions & Cyclohexenone 4 & Conditions & Product 5 \\
\hline 2 & $1 \mathrm{a}$ & $\begin{array}{l}\text { (1). DABCO (2.0 equiv.) } \\
\text { aq. THF, r, } 15 \text { min. } \\
\text { (2). } 2,4-\text { pentanedione (1.1 equiv.) } \\
\text { rt, } 24 \mathrm{~h}, 3 \mathrm{a} \text { ( } 44 \% \text { ) } \\
\text { (3). } \mathrm{K}_{2} \mathrm{CO}_{3}(1.1 \text { equiv.) } \\
\text { EtOH, reflux, } 30 \mathrm{~min} \text {. }\end{array}$ & $4 a(72 \%)$ & $\begin{array}{l}\mathrm{I}_{2}(1.1 \text { equiv. }) \\
\mathrm{MeOH}, 40-50{ }^{\circ} \mathrm{C} \\
5 \mathrm{~h} \\
\mathrm{I}_{2}(1.1 \text { equiv. }) \\
\text { EtOH, 40-50 }{ }^{\circ} \mathrm{C} \\
12 \mathrm{~h}\end{array}$ & $\begin{array}{l}\mathrm{CH}_{3} \\
\mathrm{Ca}(\mathrm{R}=\mathrm{Me}, 84 \%) \\
5 \mathrm{~b}(\mathrm{R}=\mathrm{Et}, 77 \%)\end{array}$ \\
\hline 4 & $1 b$ & $\begin{array}{l}\text { (1). DABCO (2.0 equiv.) } \\
\text { aq. THF, rl, } 15 \text { min. } \\
\text { (2). } 2,4-\text { pentanedione ( } 1.1 \text { equiv.) } \\
\text { t.. } 24 \mathrm{~h}, 3 \mathrm{~b}(25 \%) \\
\text { (3). } \mathrm{K}_{2} \mathrm{CO}_{3}(1.1 \text { equiv.) } \\
\text { EtOH, reflux, } 30 \text { min. }\end{array}$ & 6) & $\begin{array}{l}\mathrm{I}_{2}(1.1 \text { equiv. }) \\
\mathrm{MeOH}, 40-50{ }^{\circ} \mathrm{C} \\
3 \mathrm{~h} \\
\mathrm{I}_{2}(1.1 \text { equiv. }) \\
\mathrm{E} 1 \mathrm{OH}, 40-50^{\circ} \mathrm{C} \\
12 \mathrm{~h}\end{array}$ & $\begin{array}{l}\mathrm{OR} \\
\mathrm{sc}(\mathrm{R}=\mathrm{Me}, 88 \%) \\
\mathbf{5 d}(\mathrm{R}=\mathrm{Et}, 75 \%)\end{array}$ \\
\hline 6 & $1 c$ & $\begin{array}{l}\text { (1). DABCO (2.0 equiv.) } \\
\text { aq. THF, } \mathrm{r}, 15 \mathrm{~min} \text {. } \\
\text { (2). } 2,4-\text { pentanedione ( } 1.1 \text { equiv.) } \\
\text { it, } 24 \mathrm{~h}, 3 \mathrm{c}(45 \%) \\
\text { (3). } \mathrm{K}_{2} \mathrm{CO}_{3}(1.1 \text { equiv.) } \\
\text { EtOH, reflux, } 30 \text { min. }\end{array}$ & 4c (59\%) & $\begin{array}{l}\mathrm{I}_{2}(1.1 \text { equiv. }) \\
\mathrm{MeOH}, 40-50^{\circ} \mathrm{C} \\
4 \mathrm{~h} \\
\mathrm{I}_{2}(1.1 \text { equiv. }) \\
\text { EtOH, 40-50 }{ }^{\circ} \mathrm{C} \\
12 \mathrm{~h}\end{array}$ & $\begin{array}{l}5 \mathrm{e}(\mathrm{R}=\mathrm{Me}, 78 \%) \\
5 \mathrm{f}(\mathrm{R}=\mathrm{Et}, 70 \%)\end{array}$ \\
\hline
\end{tabular}

5b, 5 d, and 5 f. When we used etlanol, somewhat longer reaction time was required.

In sumnary, we have developed an efficient synthetic methodology for the synthesis of 4-methylene-2-cyclohexenones, 4-methoxymethyl anisoles, and 4-cthoxymethyl phenetoles in moderate yields, starting from the BaylisHillman acetates.

\section{Experimental Section}

Typical procedure for the synthesis of 3a. To a stirred solution of the Baylis-f Iillman acetate (1a. $436 \mathrm{mg}, 2 \mathrm{mmol}$ ) in aq. THF $\left.\left(\mathrm{THF} / \mathrm{H}_{2} \mathrm{O}\right)=3: 1,10 \mathrm{~mL}\right)$ was added $\mathrm{DABCO}$ (448 $\mathrm{mg}, 4 \mathrm{mmol}$ ) and stirred at room temperature for 15 min. To the reaction mixture 2.4-pentanedione $(220 \mathrm{mg}, 2.2$ mmol) was added and stirred at room temperature for $24 \mathrm{~h}$. $\Lambda$ fter the normal aqueous workup and column chromatographic purification process (hexanes/ether, $4: 1$ ) pure 3a was obtained, $228 \mathrm{mg}(44 \%)$. Spectroscopic data of prepared compounds are as follows. 3a: 44\%: 'Il NMR (CDCl $\delta$ $1.90(\mathrm{~s}, 3 \mathrm{H}), 2.15(\mathrm{~s}, 3 \mathrm{H}), 2.26(\mathrm{~s}, 3 \mathrm{H}), 4.56(\mathrm{~d} . J=12.6 \mathrm{~Hz}$, 1H I). 4.90 (d. $J=12.6[\mathrm{lz}, \mathrm{II}]), 5.93$ (s, 1HI). 6.13 (s, II]), $7.17-7.27(\mathrm{~m}, 5 \mathrm{H}) .3 \mathrm{~b}: 25 \% ;{ }^{1} \mathrm{H}$ NMR $\left(\mathrm{CDCl}_{3}\right) \delta 1.94(\mathrm{~s}$, $3 \mathrm{H}$ ). $2.14(\mathrm{~s}, 3 \mathrm{I}) .2 .26$ (s. $3 \mathrm{H}$ ). 4.55 (d. $J=12.6 \mathrm{Ilz}$. IIJ), $4.88(\mathrm{~d} . J=12.6 \mathrm{~Hz}, \mathrm{lH}), 5.94(\mathrm{~s}, \mathrm{lH}), 6.15(\mathrm{~s}, \mathrm{lH}), 7.14-$ $7.30\left(\mathrm{~m} .4 \mathrm{l}\right.$ ). 3c: $45 \%$; ' [ ] NMR ( $\left.\mathrm{CDCl}_{3}\right) \delta 0.98$ (t. $J=6.6$ $\mathrm{Hz}, 3 \mathrm{H}), \mathrm{I} .89$ (s, $3 \mathrm{H}), 2.15$ (s, 3H), 2.50-2.70 (m, 2H), 4.57 $(\mathrm{d}, J=12.6][\mathrm{z}, \mathrm{I}] \mathrm{]}), 4.89(\mathrm{~d}, J=[2.6][\mathrm{z}, 1][) .5 .86(\mathrm{~s} . \mathrm{II})$, $6.09(\mathrm{~s}, \mathrm{lH}), 7.15-7.28(\mathrm{~m}, 5 \mathrm{H}){ }^{13} \mathrm{C}$ NMR (CDC1 $\left.1_{3}\right) \delta 8.04$,
$28.29,30.60,31.39,45.07,73.41,123.54,127.18,128.24$ $128.68,138.98,148.52,201.28,202.64,202.80$.

Typical procedure for the synthesis of $4 a$. To a stirred solution of 3a (129 mg, $0.5 \mathrm{mmol})$ in ethanol $(5 \mathrm{~mL})$ was added $\mathrm{K}_{2} \mathrm{CO}_{3}$ (76 mg. $0.55 \mathrm{mmol}$ ) and heated to reflux for 30 min. After the normal aqueous workup and column chromatographic purification process (hexanes/ether, $10: 1$ ) pure 4a was obtained, $72 \mathrm{mg}(72 \%)$. 4a: $72 \%$ : IR (KBr) 1666, 1585, $1496 \mathrm{~cm}$ '; 'H NMR (CDCl $) \delta 2.11(\mathrm{~d}, j=1.5$ H[z, 3[]), $2.77(\mathrm{dd}, f=16.1$ and $5.5 \mathrm{Ilz}, 1] \mathrm{l}), 2.91$ (dd. $J=$ 16. $\mathrm{l}$ and $9.3 \mathrm{~Hz}$. IH) $4.0 \mathrm{l}-4.06(\mathrm{~m}, \mathrm{lH}) .5 .06(\mathrm{app} \mathrm{t}, J=1.5$ H[z, II]), 5.5I (s, 1]1), 6.00(s, 1][), 7.19-7.36 (m, 5[]): ${ }^{1.3} \mathrm{C}$ N.MR $\left(\mathrm{CDCl}_{3}\right) \delta 20.62,43.74,46.81,117.99,127.05$, $127.86,127.88,128.61,141.29,146.05,154.08,198.35,4 \mathbf{b}$ : $60 \%$; white solid mp 99- $1000^{\circ} \mathrm{C}$; IR ( $\left.\left.\mathrm{KBr}\right)\right] 658,1585,1489$ $\mathrm{cm}^{-1} ;{ }^{1}$ ] [ NMR $\left.\left(\mathrm{CDCl}_{3}\right) \delta 2 . \mathrm{l}\right](\mathrm{d} . J=1.2 \mathrm{Ilz}, 3 \mathrm{I}), 2.75(\mathrm{dd}$ $J=16.1$ and $5.6 \mathrm{~Hz}, \mathrm{lH}) .2 .86(\mathrm{dd}, J=16.1$ and $9.0 \mathrm{~Hz}, \mathrm{lH})$, $3.99-4.04(\mathrm{~m}, 1 \mathrm{H}), 5.06($ app t. $J=1.6 \mathrm{H}[\mathrm{z}, 1 \mathrm{H}), 5.26(\mathrm{~s} . \mathrm{IJ})$, $5.99(\mathrm{~s}, \mathrm{JH}) .7 .14(\mathrm{~d}, J=8.4 \mathrm{~Hz}, 2 \mathrm{H}) .7 .30(\mathrm{~d}, J=8.4 \mathrm{~Hz}$ 2]1): ${ }^{1.3} \mathrm{C}$ NMR $\left.(\mathrm{CDC}]_{3}\right) \delta 20.56,43.55,46.15,118.12$, 127.86, 128.72, I29.14, 132.79, 139.72, 145.57, 153.90, 197.87. 4c: 59\%; IR (KBr) 1666, $1496 \mathrm{~cm}^{-1}$; ] ] NMR $\left(\mathrm{CDCl}_{3}\right) \delta \mathrm{l} .15(\mathrm{t}, J=7.5 \mathrm{~Hz}, 3 \mathrm{H}), 2.46(\mathrm{qd}, J=7.5$ and 1.2 I[z, 2[]$), 2.78(\mathrm{dd}, J=16.2$ and $5.5 \mathrm{Ilz}, 1] \mathrm{l}), 2.93$ (dd. $J=$ 16.2 and $9.1 \mathrm{~Hz} . \mathrm{IH}), 4.00-4.05(\mathrm{~m}, \mathrm{lH}) .5 .06(\mathrm{app} \mathrm{t} . J=1.2$ H[z, I]I) 5.53 (s, I]I), 5.99 (s, I][), 7.19-7.35 (m, 5l]): ${ }^{1.3} \mathrm{C}$ N.VR (CDCl $) \delta$ I l.36. 25.29, 42.66, 46.23, l l6.04, 124.7l, 126.00, 126.83, 127.55, 140.16. 144.25. 158.38, 197.67.

Typical procedure for the synthesis of 5 a. $\Lambda$ stirred 
solution of 4a (99 mg. $0.5 \mathrm{mmol}$ ) and iodine ( $140 \mathrm{mg} .0 .55$ mmol) in methanol $(3 \mathrm{~mL})$ was heated to $40-50^{\circ} \mathrm{C}$ during 5 h. After the normal aqueous workup and column chromatographic purification process (hexanes/ether, 20:1) pure $\mathbf{5 a}$ was obtained, $102 \mathrm{mg}(84 \%)$.

5a: $84 \%$; mp 56-58 ${ }^{\circ} \mathrm{C}:{ }^{1} \mathrm{H}$ NMR $\left(\mathrm{CDCl}_{3}\right) \delta 2.45$ (s, 3H). $3.28(\mathrm{~s}, 3 \mathrm{H}) .3 .78(\mathrm{~s}, 3 \mathrm{H}) .4 .19(\mathrm{~s}, 2 \mathrm{H}) .6 .67(\mathrm{~d} . J=2.7 \mathrm{~Hz}$. $\mathrm{lH}), 6.77(\mathrm{~d} . J=2.7 \mathrm{~Hz} . \mathrm{lH}), 7.34-7.42(\mathrm{~m} .5 \mathrm{H}):{ }^{13} \mathrm{C}$ NMR $\left(\mathrm{CDCl}_{3}\right) \delta 19.59,55.17$. 57.81. 69.32. 112.52. 115.47. 126.07. 126.98, 127.88, 129.26, 140.52, 141.65, 144.82. 158.60; Mass $(70 \mathrm{eV}) m z$ (rel intensity) $165(28), 196(43)$. $211(100), 242\left(\mathrm{M}^{+} .57\right)$

5b: $77 \% ;{ }^{1} \mathrm{H}$ NMR $\left(\mathrm{CDCl}_{3}\right) \delta 1.20(\mathrm{t}, J=7.2 \mathrm{~Hz}, 3 \mathrm{H})$. 1.38 (t. $J=7.2 \mathrm{~Hz}, 3 \mathrm{H}$ ). 2.45 (s, $3 \mathrm{H}) .3 .42(\mathrm{q}, J=7.2 \mathrm{~Hz}$. $2 \mathrm{H}), 4.02(\mathrm{q}, J=7.2 \mathrm{~Hz}, 2 \mathrm{H}), 4.23$ (s. $2 \mathrm{H}), 6.66(\mathrm{~d}, J=2.7$ $\mathrm{Hz}, 1 \mathrm{H}$ ). $6.76($ d. $J=2.7 \mathrm{~Hz} .1 \mathrm{H}), 7.33-7.44(\mathrm{~s}, 5 \mathrm{H}) ;{ }^{13} \mathrm{C}$ NMR $\left(\mathrm{CDCl}_{3}\right) \delta 14.82 .15 .27 .19 .65,63.34,65.50,67.33$. 113.24 . 116.14, 126.10, 126.91. 127.82, 129.32, 140.43. 141.77. 144.73, 157.95: Mass (70 eV) $m z$ (rel intensity) 197 (57). $225(100) .241$ (38). $270\left(\mathrm{M}^{+} .83\right)$

5c: $88 \%$ : ${ }^{1} \mathrm{H}$ NMR $\left(\mathrm{CDCl}_{3}\right) \delta 2.45(\mathrm{~s}, 3 \mathrm{H}) .3 .30(\mathrm{~s}, 3 \mathrm{H})$. 3.79 (s. $3 \mathrm{H}$ ) 4.15 (s. $2 \mathrm{H}$ ). 6.62 (d. $J=2.6 \mathrm{~Hz} .1 \mathrm{H}$ ). 6.77 (d. $J$ $=2.6 \mathrm{~Hz}, \mathrm{lH}), 7.32-7.40(\mathrm{~m}, 4 \mathrm{H}) ;{ }^{13} \mathrm{C}$ NMR $\left(\mathrm{CDCl}_{3}\right) \delta$ $18.60,54.21$. 56.93, 68.25. 111.53, 114.61, 125.03. 127.08. 129.59. 132.14, 139.04, 139.69. 142.58, 157.70: Mass (70 eV) $m z$ (rel intensity) 195 (37), 209 (90), 245 (100). 276 $\left(\mathrm{M}^{+}, 77\right), 278\left(\mathrm{M}^{+}+2.27\right)$.

5d: $75 \%,{ }^{1} \mathrm{H}$ NMR $\left(\mathrm{CDCl}_{3}\right) \delta 1.2 \mathrm{l}(\mathrm{t}, J=6.9 \mathrm{~Hz}, 3 \mathrm{H})$. 1.39 (t. $J=6.9 \mathrm{~Hz}, 3 \mathrm{H}$ ). $2.44(\mathrm{~s}, 3 \mathrm{H}$ ). 3.44 (q. $J=6.9 \mathrm{~Hz}$. $2 \mathrm{H}), 4.02(\mathrm{q}, J=6.9 \mathrm{~Hz}, 2 \mathrm{H}), 4.19(\mathrm{~s} .2 \mathrm{H}), 6.61(\mathrm{~d}, J=2.4$ Hz, IH). 6.76 (d. $J=2.4 \mathrm{~Hz} .1 \mathrm{H}) .7 .36(\mathrm{~s}, 4 \mathrm{H}) ;{ }^{13} \mathrm{C}$ NMR $\left(\mathrm{CDCl}_{3}\right) \delta 14.80 .15 .28 .19 .64 .63 .38 .65 .63 .67 .26 .113 .24$. 116.24. 126.03, 128.00, 130.62. 133.06, 140.15, 140.59. 143.48. 158.04; Mass (70 eV) $\mathrm{mz}$ (rel intensity) $196(47)$. $223(70) .23 \mathrm{I}(43) .259(100) .304\left(\mathrm{M}^{+} .70\right) .306\left(\mathrm{M}^{-}+2.23\right)$

5e: $78 \%:{ }^{1} \mathrm{H} \mathrm{NMR}\left(\mathrm{CDCl}_{3}\right) \delta 1.30(\mathrm{t} . J=7.5 \mathrm{~Hz} .3 \mathrm{H}) .2 .80$ (q. $J=7.5 \mathrm{~Hz} .2 \mathrm{H}), 3.27$ (s. $3 \mathrm{H}), 3.8 \mathrm{l}(\mathrm{s}, 3 \mathrm{H}), 4.19$ (s, $2 \mathrm{H})$. $6.67(\mathrm{~d} . J=2.7 \mathrm{~Hz} . \mathrm{lH}) .6 .82(\mathrm{~d}, J=2.7 \mathrm{~Hz} . \mathrm{lH}) .7 .35-7.44$ (m. $5 \mathrm{H}):{ }^{13} \mathrm{C} \mathrm{NMR}\left(\mathrm{CDCl}_{\hat{j}}\right) \delta 15.56,25.87,55.23,57.79$. 68.83, 112.43, 113.89. 125.55. 127.00. 127.88. 129.35. 141.87. 145.11. 146.49, 158.89: Mass (70 eV) $m z$ (rel intensity) $165(37) .195(55), 224(100), 256\left(\mathrm{M}^{-}, 61\right)$.

5f: $70 \%:{ }^{\circ} \mathrm{H}$ NMR $\left(\mathrm{CDCl}_{3}\right) \delta 1.19(\mathrm{t} . J=6.9 \mathrm{~Hz}, 3 \mathrm{H}), 1.30$ (t. $J=7.5 \mathrm{~Hz} .3 \mathrm{H}$ ). 1.40 (t. $J=6.9 \mathrm{~Hz} .3 \mathrm{H}$ ). 2.80 (q. $J=7.5$
Hz. $2 \mathrm{H}), 3.4 \mathrm{I}(\mathrm{q} . J=6.9 \mathrm{~Hz}, 2 \mathrm{H}) .4 .03$ (q. $J=6.9 \mathrm{~Hz} .2 \mathrm{H}$ ), $4.23(\mathrm{~s}, 2 \mathrm{H}) .6 .66(\mathrm{~d} . J=2.7 \mathrm{~Hz} . \mathrm{lH}) .6 .81(\mathrm{~d}, J=2.7 \mathrm{~Hz}$, 1H). $7.31-7.45(\mathrm{~m} .5 \mathrm{H}):{ }^{13} \mathrm{C}$ NMR $\left(\mathrm{CDCl}_{3}\right) \delta 14.84,15.28$. $15.50,25.87 .63 .33 .65 .46 .66 .79,113.07$. 114.52. 125.55, $126.88,127.76,129.37,141.96,144.97,146.36,158.20$ : Mass $(70 \mathrm{eV}) m z$ (rel intensity) 165 (41). 183 (43). 238 (100), $255(30), 284\left(\mathrm{M}^{+} .55\right)$.

Acknowledgments. This study was financially supported by research fund of Chonnam National University in 2003. Spectroscopic data was obtained from the Korea Basic Science Institute. Kwangiu branch.

\section{References and Notes}

1. (a) Kim. T. M.: Lee. K. Y.: Kim. T. H.: Kim. T. N. Bull. Korean Chent Soc. 2003. 24. 999. (b) Kim. J. M.: Lee. K. Y.: Kim. I. N. Bull. Korem Chem. Soc. $2003,24,1057$.

2. Chamakh. A.: Amri, H. Tetrahedron Lett. 1998, 39.375.

3. For the selective introduction of nucleophiles at the secondary berizylic position of the Baylis-Hillman acetates via the corresponding DABCO salts. see: (a) Im. Y. J.: Kim. J. M.: Mun1. J. H.: Kim. J. N. Bull Korean Chent. Soc. 2001. 22. 349. (b) Basavaiah. D.; Kumaragurubaran. N.: Sharada, D. S. Tetrahedron Lett. 2001, 42.85. (c) Basavaiah. D.: Rao. A. J.; Satyanarayana. T. Chem. Rev 2003. 103,811 and further references cited therein.

4. Dorothea. G. Phenol Derivatives. Llhmams Encyclopedia of Industrial Chentistr: Barbara. E.: Stephen. H.: Gail. S.. Eds.: VCH: Weinheim. 1991: Vol. Al9.

5. For the synthetic applications of the $p$-methoxymethyl anisole. see: (a) Oikawa. Y; Nishi, T.: Yonemitsu. O. Tetrahedron Lett. 1983. 24. 4037. (b) Fellows. I. M.: Kaelin. D. E.. J.: Martin, S. F. 1. Am. Chem Soc. 2000. 122. 10781 . (c) Kanazawa. A. M: Denis. J.-N.: Greene. A. E. J. Org. Chent 1994. 59. 1238. (d) Silva. C. B.-D: Pale. P. Tetrahedron: Astmmetry 1998. 9. 3951. (e) Oikawa, Y; Nishi. T; Yonemitsu, O. J. Chent Soc. Perkin Trans. I 1985.7

6. Unpublished results. The results on the synthesis of dihydropyran derivatives will be published in due course.

7. For the synthesis of methylene cyclohesenones. see: (a) Davis. B. R.: Johnsont. S. J. J. Chent. Soc., Perkin Trans. I 1979. 2840. (b) Jung. M. E.: Ravle. H. L. Swmh. Conmmin. 1994. 2H, 197.

8. 4-Methylene 2-cyclohexenones $\mathbf{t b}$ and $\mathbf{t c}$ could also be synthesized by similar method (in situ generation of DABCO salt in ethanol and the following reaction with 2.4-pentanedione in the presence of $\mathrm{K}_{2} \mathrm{CO}_{3}$ ) in 41 and $40^{\circ}$. 0 . respectively.

9. For the aromatization reaction of cyclohexenone derivatives with iodine and alcohol system, see: (a) Tamura, Y: Yoshimoto, Y. Chem. hid. 1980, 888. (b) Kotnis. A. S. Tetrahedron Lett. 1990. 31. $48 \mathrm{l}$ and further references cited in reference la. 